\title{
Phish: A Novel Hyper-Optimizable Activation Function
}

This paper was downloaded from TechRxiv (https://www.techrxiv.org).

\section{LICENSE}

CC BY 4.0

SUBMISSION DATE / POSTED DATE

$18-12-2021 / 19-01-2022$

\section{CITATION}

Naveen, Philip (2021): Phish: A Novel Hyper-Optimizable Activation Function. TechRxiv. Preprint. https://doi.org/10.36227/techrxiv.17283824.v2

$\mathrm{DOI}$ 


\title{
Phish: A Novel Hyper-Optimizable Activation Function
}

\author{
Philip Naveen
}

January 15, 2022

\begin{abstract}
Deep-learning models estimate values using backpropagation. The activation function within hidden layers is a critical component to minimizing loss in deep neural-networks. Rectified Linear (ReLU) has been the dominant activation function for the past decade. Swish and Mish are newer activation functions that have shown to yield better results than ReLU given specific circumstances. Phish is a novel activation function proposed here. It is a composite function defined as $\mathrm{f}(\mathrm{x})=\mathrm{xTanH}(\operatorname{GELU}(\mathrm{x}))$, where no discontinuities are apparent in the differentiated graph on the domain observed. Generalized networks were constructed using different activation functions. SoftMax was the output function. Using images from MNIST and CIFAR-10 databanks, these networks were trained to minimize sparse categorical crossentropy. A large scale cross-validation was simulated using stochastic Markov chains to account for the law of large numbers for the probability values. Statistical tests support the research hypothesis stating Phish could outperform other activation functions in classification. Future experiments would involve testing Phish in unsupervised learning algorithms and comparing it to more activation functions.
\end{abstract}

\section{Introduction}

Deep-learning algorithms are capable of solving complex problems. They use a series of synaptic weights and perceptrons to mimic the human thinking process. The success of training deep neuralnetworks (DNN) relies much on the activation function used in them. In each perceptron, two phases occur: a summation and transformation. In the summation, the inputs are multiplied with synaptic weights, which are initially generated at random, with a Hadamard product (Silaparasetty, 2020). The transformation step consists of the summated vector being parsed through an activation function in addition to an optional bias (Sharma, Sharma, and Athaiya, 2020). Early architectures used TanH and Sigmoid extensively. However, the more complex DNNs required better activation functions.

The most commonly used activation function in DNNs is Rectified Linear (ReLU) Agarap, 2018). It is a less probability inspired piecewise function with no discontinuities. It has a jump discontinuity when differentiated due to the sharp turn at the origin. Experiments demonstrated that ReLU increased the performance in DNNs, outperforming TanH (Abdelouahab, Pelcat, and Berry, 2017) and Sigmoid (Pratiwi, Windarto, Susliansyah, Aria, Susilowati, Rahayu, Fitriani, Merdekawati, and Rahadjeng, 2020). However, ReLU has some faults. One of the biggest ones is the dying ReLU issue, but luckily leaky ReLU partially solved this issue via augmenting the negative domain of the function (Dubey and Jain, 2019).

Swish and Mish are newer activation functions that have recently gained traction (Ramachandran, Zoph, and Le, 2017). They are both composite and comprise at least one existing activation function. Unlike ReLU, these functions are non-linear, and their derivatives are void of discontinuities. They both perpetually increase and pass through the origin $(0,0)$. The new activation function created here would follow the parameters of Swish and Mish (Misra, 2020).

A new activation function named Phish is proposed here. Phish is defined as $\mathrm{f}(\mathrm{x})=\mathrm{xTanH}(\operatorname{GELU}(\mathrm{x}))$. Phish is non-monotonic, unlike other activation functions where the slope is completely positive. On the interval from $[0, \infty]$, it is completely positive and passes through the origin $(0,0)$. Phish estimates updates in backpropagation using the concepts of continuity, differentiability, and nonmonotonicity.

An experimental simulation to compare Phish to existing functions will be conducted. The levels of independent variable will be GELU, Swish, TanH, Sigmoid, ReLU, Mish, and Phish. There was no control. The dependent variable was the minimization of sparse categorical crossentropy (SCC), 
which is one of the most common loss functions in classification. Several constants variables will be held, such as the DNN layers, optimizer, output function, and learning rate.

\section{Phish Activation Function}

\subsection{Backpropagation and Update Gradients}

To calculate the update gradient, the rate of change in loss $\mathrm{L}$ must be determined. Theoretically, though impractical, this can be determined via calculating the slope between two datapoints with an infinitesimal distance. The standard error can be approximated via finding the instantaneous rate of change in loss (eg. determining a partial derivative in respect to $\mathrm{z}$ ). When

$$
\frac{\Delta L}{\Delta z_{k}^{[l]}} \approx \frac{\partial L}{\partial z_{k}^{[l]}}
$$

the calculated error can be propagated to every weight in the neural-network. Using the weighted input, loss derivative, and activation function derivative, the update gradient can be calculated using basic algebra such that

$$
\frac{\Delta L}{\Delta z_{k}^{[l]}}=\frac{\partial L}{\partial z^{[l-1]}}=\frac{\partial L}{\partial z^{[l]}} A^{[l-1]^{\prime}}\left(z^{[l-1]}\right)
$$

across many iterations. Due to space constraints, optimization and further analysis of partial derivatives has been omitted. As can be seen, the activation function and its derivative are critical in the training of deep neural-networks (DNNs) in supervised classification, or in unsupervised classification (eg. discriminators in generative adversarial networks). Substituting various activation functions can vastly alter the minimization of loss.

\subsection{Derivation and Implementation}

Much like Mish, Phish is a composite function. It comprises two existing activation functions, those being TanH and GELU. The inner function GELU 11 is defined as

$$
\operatorname{GELU}(x)=\frac{x}{2}\left[1+\frac{2}{\sqrt{\pi}} \int_{0}^{\frac{x}{\sqrt{2}}} e^{-t^{2}} d t\right]
$$

to approximate ReLU

$$
\operatorname{ReLU}(x)=x^{+}=\max (0, x)=\left\{\begin{array}{l}
x \text { if } x>0 \\
0 \text { if } x<0
\end{array}\right.
$$

such that no discontinuities occur on the differentiated graph. ReLU is perhaps the most used activation function in DNNs. It has shown to be effective in large-scale classification problems, often used in image classification.

The outer activation function $\mathrm{TanH}^{2}$, is is defined by

$$
\operatorname{TanH}(x)=\frac{e^{x}-e^{-x}}{e^{-x}+e^{x}}
$$

Since Phish is expressed in terms of other equations and variables, the true form of the equation can be determined. Therefore, through substituting variables and rearranging the terms, the Phish equation in the most pure form can be defined as

$$
\operatorname{Phish}(x)=x(\text { TanH } \circ \text { GELU })=x \frac{e^{\frac{x}{2}\left[1+\frac{2}{\sqrt{\pi}} \int_{0}^{\frac{x}{\sqrt{2}}} e^{-t^{2}} d t\right]}-e^{-\frac{x}{2}\left[1+\frac{2}{\sqrt{\pi}} \int_{0}^{\frac{x}{\sqrt{2}}} e^{-t^{2}} d t\right]}}{e^{-\frac{x}{2}\left[1+\frac{2}{\sqrt{\pi}} \int_{0}^{\frac{x}{\sqrt{2}}} e^{-t^{2}} d t\right]}+e^{\frac{x}{2}\left[1+\frac{2}{\sqrt{\pi}} \int_{0}^{\frac{x}{\sqrt{2}}} e^{-t^{2}} d t\right]}}
$$

\footnotetext{
${ }^{1}$ GELU is an approximation of the ReLU activation function defined as The main implementation of such a function is to avoid the large jump discontinuity apparent in ReLU, which occurs at the origin $(0,0)$ on the Cartesian coordinate system. The non-linear function seems to outperform ReLU and ELU in certain tasks in language processing and classification.

${ }^{2}$ TanH is the analogue hyperbolic tangent function often used throughout trigonometry. Similar in concept to Sigmoid, it has two horizontal asymptotes. However, these exist at $\mathrm{y}= \pm 1$, which indicates that the domain is half negative. Therefore, $\mathrm{TanH}_{+}$exists only rightward of the origin $(0,0)$, which it crosses.
} 
Using the backpropagation equation derived in the introduction, the activation function $\mathrm{A}(\mathrm{x})$ can be substituted with any activation function to simulate the calculation of update gradients. Such gradients for Phish require its derivative. Update gradient calculation can be formulated via substituting the Phish derivative yielded from equation 9 using the chain rule.

$$
\therefore \frac{\Delta L}{\Delta z_{k}^{[l]}}=\frac{\partial L}{\partial z^{[l-1]}}=\frac{\partial L}{\partial z^{[l]}} \operatorname{Phish}^{[l-1]^{\prime}}\left(z^{[l-1]}\right)
$$

Based on the assumption that

$$
\operatorname{erf}(z)=\frac{2}{\pi} \int_{0}^{z} e^{-t^{2}} d t
$$

where $\mathrm{z}$ is any complex number, the derivative can be calculated by substituting integrals from equation 8 , rearranging the terms, and applying the chain rule and fundamental theorem of calculus onto all sides in equation 9.

$$
\therefore \frac{\Delta L}{\Delta z_{k}^{[l]}}=\frac{\partial L}{\partial z^{[l-1]}} \times \frac{\mathrm{d}}{\mathrm{d} z^{[l-1]}}\left[z^{[l-1]} \frac{e^{z^{[l-1]}\left(\operatorname{erf}\left(\frac{z^{[l-1]}}{\sqrt{2}}\right)+1\right)-1}}{e^{z^{[l-1]}\left(\operatorname{erf}\left(\frac{z^{[l-1]}}{\sqrt{2}}\right)+1\right)+1}}\right]
$$

For the purpose of conciseness, the full algebra of the Phish derivative was omitted from this paper. However, in terms of itself, it exists as

$$
\operatorname{Phish}^{\prime}\left(z^{[l-1]}\right)=\frac{\operatorname{Phish}\left(z^{[l-1]}\right)}{z^{[l-1]}}+z^{[l-1]} \operatorname{TanH}^{\prime}\left(\operatorname{GELU}\left(z^{[l-1]}\right)\right) \operatorname{GELU}^{\prime}\left(z^{[l-1]}\right)
$$

\section{Evaluation}

A simulation was conducted to compare activation functions. The levels of independent variable were GELU, Swish, TanH, Sigmoid, ReLU, Mish, and Phish. The minimization of loss was studied using DNNs.

One Intel i7 computer was obtained. Python3 was installed onto the machine with machinelearning and linear algebra dependencies. Custom computer code was developed to test and compare Phish. For the procedure, 170,000 training and 50,000 testing images were gathered. The images were preprocessing via normalization and cropping. The preprocessing was limited to generalize the training process.

A generic neural-network was fabricated for testing. The parameters, matrix multiplication, and training dynamics are located in the appendix.

$$
J(w)=\frac{-1}{n} \sum_{j=1}^{n}\left[y_{j} \log \left(\widehat{y_{j}}\right)+\left(1-y_{j}\right) \log \left(1-\widehat{y}_{j}\right)\right]
$$

was substituted with SCC.

$$
\therefore J(w)=-\sum_{j=1}^{n} y_{j} \log \left(\widehat{y_{j}}\right)
$$

where $\mathrm{w}$ represents the arbitrary parameters of a given network with the y values representing the predicted and true labels. This was done so the network would assume correct classifications can only be a single prediction.

SoftMax was used for the output layer. It is a deep-learning probability distribution function used in multi-class identification problems. It is

$$
\sigma(\vec{z})_{i}=\frac{e^{z_{i}}}{\sum_{j=1}^{K} e^{z_{j}}}
$$

where the input and output functions of the network calculate for the input vector. During testing, $\mathrm{K}=10$ was constant because each of the databanks used had ten possible labels. 
The levels of independent variable were tested. This was done twenty-five separate times for each activation function. The minimization of SCC was recorded.

This project was conducted on a laptop without graphics processing units or cloud servers. Therefore, a large scale cross-validation was not reasonable. A memoryless stochastic model was more favorable for such a purpose. Thus a Markov chain was developed to simulate the process. The combination of the two methods calculated the loss.

Graph 1: The Effect of Activation Functions on Minimizing Sparse Categorical Crossentropy

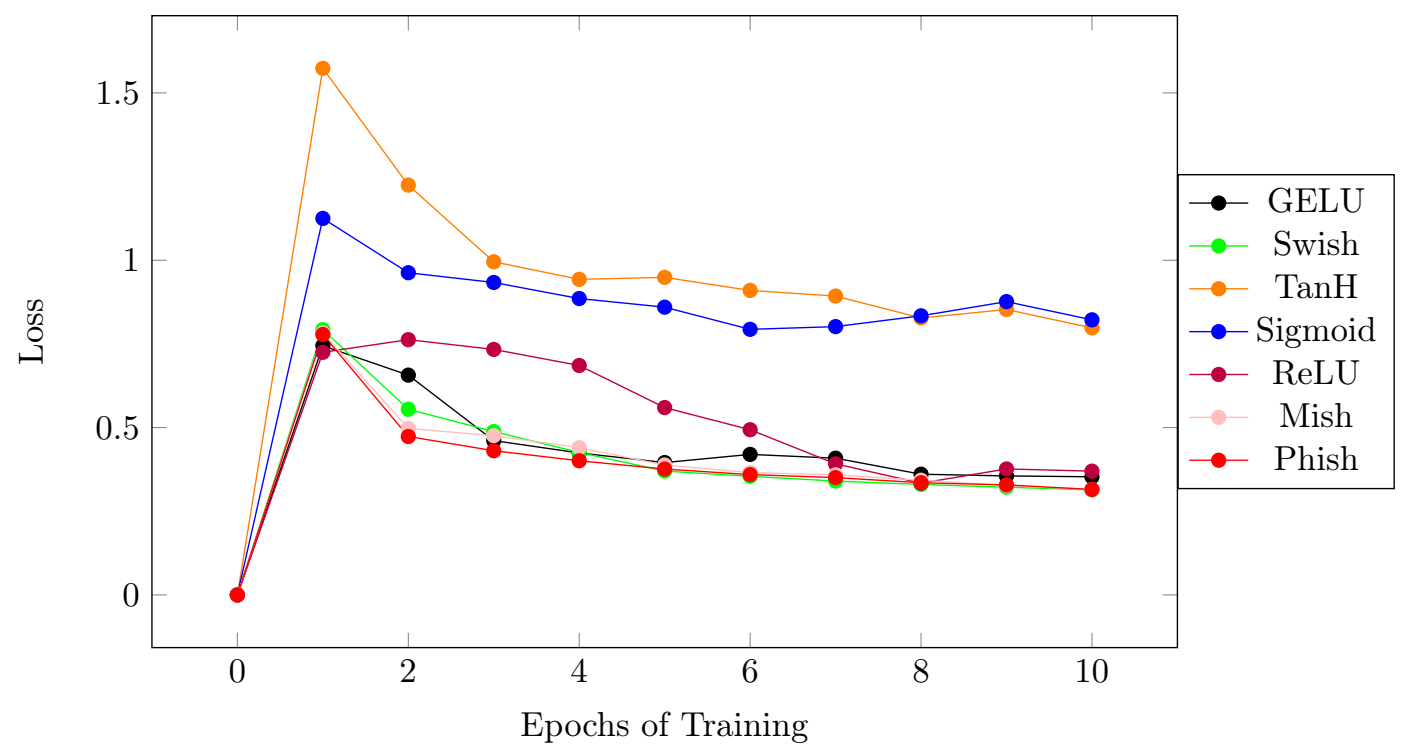

The effect of activation functions on minimizing the loss in classification for DNNs was tested. Various datasets were used to simulate classification backpropagation. GELU, Swish, TanH, Sigmoid, ReLU, Mish, and Phish can be seen in graph 1.

This particular graph shows the trend when training on MNIST fashion. The graph was the average loss across epochs calculated from twenty-five trials. Across the various epochs, it can be seen that Phish and Swish had a similar minimization of SCC. TanH and Sigmoid had significantly lower reduction of loss compared to Swish and Phish. From the data collected it could be inferred that

Similar patterns were apparent when the networks trained on MNIST numbers and CIFAR-10 image databanks. Phish consistently outperformed TanH and Sigmoid. It was either on-par or slightly superior to Swish .It also showed similar training patterns to GELU. The results of the experiment show that Phish is a promising alternative activation function.

Table 1: Table 1: Statistical Analysis at 0.05 Significance Level under 48 Degrees of Freedom

\begin{tabular}{|l|l|l|l|}
\hline T-Test & Calculated Value & Table Value & Result \\
\hline Phish vs. GELU & 3.336 & 2.011 & Significant \\
\hline Phish vs. Swish & 1.996 & 2.011 & Not Significant \\
\hline Phish vs. TanH & 56.331 & 2.011 & Significant \\
\hline Phish vs. Sigmoid & 35.088 & 2.011 & Significant \\
\hline Phish vs. ReLU & 4.281 & 2.011 & Significant \\
\hline Phish vs. Mish & 1.782 & 2.011 & Not Significant \\
\hline
\end{tabular}

This data table shows some of the compared levels of independent variable. Independent parametric t-tests ${ }^{3}$ were calculated to determine the significance of the data collected. The value

\footnotetext{
${ }^{3}$ T-tests were a favorable method to testing for significance in this dataset. Traditionally, a one way ANOVA test could imply significance. However, that is suitable for identifying differences and patterns holistically. This experiment sought to determine whether Phish is more or less effective at loss minimization. Therefore, comparing pairs of activation functions using independent t-tests was more ideal.
} 
of significance was at 0.05 , and was granted 48 degrees of freedom. A table value of 2.011 was used. A null hypothesis was generated. It stated that there would be no difference between any of the tested activation functions when given the task of minimizing SCC.

The majority of comparisons relating to Phish were significant. In each case of significance, Phish outperformed the competing activation function. Phish showed to outperform Swish and Mish as well, but the difference was not notable such that a t-test could identify significance. Experiments with larger datasets and deeper models could be used to further investigate the relationships between Swish, Mish, and Phish in the future.

\section{Discussion}

\subsection{Procedural Flaws}

There were many sources for error in the experimentation done to determine the properties of Phish. The first was that Phish was only compared to three other activation functions. Another flaw was that only one architecture was tested for classification, where many could have been tested. Other combinations of optimizers, metrics, losses, and layers may result in different findings.In addition, a true simulation of the loss was never conducted. The simulations were partially crossvalidation, but the other component simulated sequences of predictions using stochastic markov chains.

To remedy these errors in the future, various types of classification algorithms could be tested using the activation functions. More functions could be compared as well. Lastly, better computers and cloud servers could be used to conduct the advanced simulations required to test Phish, that would otherwise impractical on a laptop.

\subsection{Future Applications}

Future applications of the activation function proposed in this research may vary. The first application would be further testing on types of datasets. MNIST and CIFAR-10 were used in this research. MNIST is a relatively simple dataset that most deep-learning models could solve (Xiao, Rasul, and Vollgraf, 2017). CIFAR-10 consists of RGB images, which requires better models to solve (Pandit and Kumar, 2020). Still, testing Phish on MNIST and CIFAR-10 only would limit knowledge on its properties. ImageNet is a public databank consisting of RGB images with an average resolution of $469 \times 387$ pixels (Russakovsky, Deng, Su, Krause, Satheesh, Ma, Huang, Karpathy, Khosla, Bernstein, Berg, and Fei-Fei, 2015). It is organized according to the WordNet hierarchy, and is often used when to test pretrained convolutional neural-networks.

Specialized layers in the networks used for testing were omitted throughout evaluation. Further testing could determine the effect of Phish on such models. Specific examples would include recurrent neural-networks. These networks were engineered to solve the vanishing gradient problem (Zaremba, Sutskever, and Vinyals, 2014). Gated recovery unit and long-short term memory algorithms are extensions of recurrent neural-networks (Hochreiter and Schmidhuber, 1997). When testing time series data, Phish could be implemented in these algorithms via substituting Sigmoid layers.

Another example of a future study would be utilizing Phish in generative adversarial networks. These algorithms comprise of two models, often multilayer perceptrons, engaging in a minimax game. The first model is the generator, which captures the distribution of a given dataset. The second one is the discriminator, which differentiates samples from the dataset and ones generated by the generator. Ideally, the loss of the discriminator would be maximized with the accuracy yielding $\frac{1}{2}$ everywhere. Testing Phish in a model with the purpose of maximizing loss would be an interesting future study Goodfellow, Pouget-Abadie, Mirza, Xu, Warde-Farley, Ozair, Courville, and Bengio, 2014). Lastly, Phish could be tested without the assumption that the aforementioned loss would be defined as in equations 13 and 14 .

\section{Conclusion}

Phish is a novel non-monotonic activation function. It delivered higher performance in MNIST and CIFAR-10 image classification than Sigmoid and and TanH. It rivals Swish in loss minimization. Its derivative is always positive rightward from the origin $(0,0)$. Phish evaluates calculations that 
increase the speed of loss minimization. Unlike ReLU, Phish is fully differentiable. Future studies could involve training generative adversarial networks with Phish and examining the performance. This project was conducted under adult approval with antivirus software. TensorFlow-Keras, PyTorch, and MXNet applications of the function can be found at the link: https://github. com/PhilipNaveen/Phish-A-Novel-Hyper-Optimizable-Activation-Function

\section{References}

Kamel Abdelouahab, Maxime Pelcat, and François Berry. Why tanh is a hardware friendly activation function for cnns. Proceedings of the 11th International Conference on Distributed Smart Cameras, 2017.

Abien Fred Agarap. Deep learning using rectified linear units (relu). ArXiv, abs/1803.08375, 2018.

Arun Kumar Dubey and Vanita Jain. Comparative study of convolution neural network's relu and leaky-relu activation functions. Lecture Notes in Electrical Engineering, 2019.

Ian J. Goodfellow, Jean Pouget-Abadie, Mehdi Mirza, Bing Xu, David Warde-Farley, Sherjil Ozair, Aaron C. Courville, and Yoshua Bengio. Generative adversarial nets. In NIPS, 2014.

Sepp Hochreiter and Jürgen Schmidhuber. Long short-term memory. Neural Computation, 9: 1735-1780, 1997.

Sean P. Meyn and Richard L. Tweedie. Markov chains and stochastic stability. In Communications and Control Engineering Series, 1993.

Diganta Misra. Mish: A self regularized non-monotonic activation function. In BMVC, 2020.

University of Auckland. Chapter 8: Markov chains. pages 149-173, 2018.

Suyesh Pandit and Sushil Kumar. Improvement in convolutional neural network for cifar-10 dataset image classification. International Journal of Computer Applications, 176:25-29, 2020.

Heny Pratiwi, Agus Perdana Windarto, Susliansyah Susliansyah, Ririn Restu Aria, Susi Susilowati, Luci Kanti Rahayu, Yuni Fitriani, Agustiena Merdekawati, and Indra Riyana Rahadjeng. Sigmoid activation function in selecting the best model of artificial neural networks. 2020.

Prajit Ramachandran, Barret Zoph, and Quoc V. Le. Swish: a self-gated activation function. arXiv: Neural and Evolutionary Computing, 2017.

Olga Russakovsky, Jia Deng, Hao Su, Jonathan Krause, Sanjeev Satheesh, Sean Ma, Zhiheng Huang, Andrej Karpathy, Aditya Khosla, Michael S. Bernstein, Alexander C. Berg, and Li FeiFei. Imagenet large scale visual recognition challenge. International Journal of Computer Vision, 115:211-252, 2015.

Siddharth Sharma, Simone Sharma, and Anidhya Athaiya. Activation functions in neural networks. 2020.

Vinita Silaparasetty. Perceptrons. Deep Learning Projects Using TensorFlow 2, 2020.

Han Xiao, Kashif Rasul, and Roland Vollgraf. Fashion-mnist: a novel image dataset for benchmarking machine learning algorithms. ArXiv, abs/1708.07747, 2017.

Wojciech Zaremba, Ilya Sutskever, and Oriol Vinyals. Recurrent neural network regularization. ArXiv, abs/1409.2329, 2014. 


\section{Appendix}

\subsection{Markov Chains}

A Markov chain is a stochastic graph model based in probability. They are favorable for simulating large networks of events because they are memoryless. Each chain yeilds a stochastic transition matrix (STM) (Meyn and Tweedie, 1993).

The Markov model on $\Omega$ results in the stochastic process $\left(X_{0}, X_{1}, X_{2}, \ldots X_{t}\right)$ in which the transition state between $\mathrm{x}$ and $\mathrm{y}$ complies with the properties

$$
X_{t} \in \Omega, \forall t
$$

and

$$
\mathbb{P}\left[X_{t+1}=y \mid X_{t}=x, X_{t-1}=x_{t-1}, \ldots X_{0}=x_{0}\right]=\mathbb{P}\left[X_{t+1}=y \mid X_{t}=x\right]=: P(x, y)
$$

In addition, the STM exists with non-negativity

$$
\forall x, y \in \Omega, P(x, y) \geq 0
$$

and stochasticity

$$
\sum_{y \in \Omega}=P(x, y)=1, \forall x \in \Omega
$$

where each row converges to 1 (of Auckland, 2018). This Markov model is continuous, with no termination node with $100 \%$ probability of returning to itself on the graph, and a $0 \%$ chance of transferring to any other stage. In addition, there is technically an appropriate start node in this graph. However, since this Markov model will be ran for extended periods of time, the law of large numbers states that the probability of the event occurring will be affected minimally from the first event, especially since there are only two possible stages in this model. Therefore, the chance of starting at either stage was 50\% always (Meyn and Tweedie, 1993).

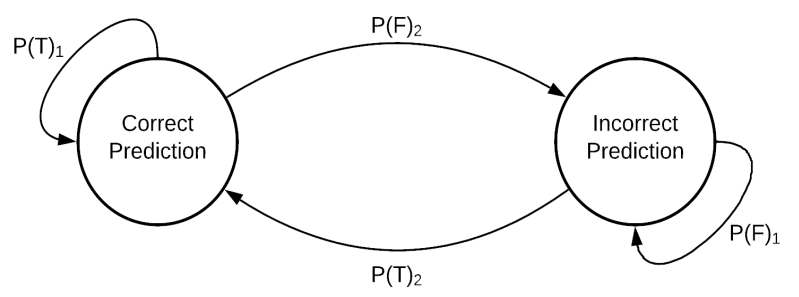

Figure 1: Markov Chain Model used for Simulating Cross-Validations for Deep Neural-Networks

The Markov chain utilized here is a two stage graph with four global locations and two local ones for each stage. An minimalistic representation of the adjacency matrix could be fabricated accordingly with $4 \times 4$ dimensions. The probability values were guaranteed using the STM

$$
\omega_{i j}=\Omega=\left[\begin{array}{ll}
P_{1}(T) & P_{2}(T) \\
P_{2}(F) & P_{1}(F)
\end{array}\right]
$$

The Markov simulation was conducted where the $P_{1}(T), P_{1}(F), P_{2}(T)$, and $P_{2}(F)$ were retrieved from a cross-validation. For each activation function, a DNN was trained across ten epochs. The prediction ratios were implanted into four graphs. Each graph was simulated for 10,000 iterations twenty-five times to follow the ideal experimental design.

\subsection{Deep Neural-Networks}

Activation functions are derived with the purpose of generating non-linearity to the inherently linear data transformed from the input layer of a neural-network. Backpropagation is the process 
where each synaptic weight in deep-learning algorithms are iteratevely finetuned to complete a task using loss calculated between the expected and actual outcomes. Suppose there is a multilayer perceptron with with weights, and biases adjusted through an arbitrary activation function $\mathrm{A}(\mathrm{x})$. In this multilayer perceptron, as with most, the weights are defined the simple matrix

$$
w^{[l]}=\left[\begin{array}{ccccc}
w_{1,1}^{[l]} & w_{1,2}^{[l]} & w_{1,3}^{[l]} & \ldots & w_{1, n}^{[l]} \\
w_{2,1}^{[l]} & w_{2,2}^{[l]} & w_{2,3}^{[l]} & \ldots & w_{2, n}^{[l]} \\
w_{3,1}^{[l]} & w_{3,2}^{[l]} & w_{3,3}^{[l]} & \ldots & w_{3, n}^{[l]} \\
w_{n^{[l]}, 1}^{[l]} & w_{n^{[l]}, 2}^{[l]} & w_{n^{[l]}, 3}^{[l]} & \ldots & w_{n^{[l]}, n^{[l-1]}}^{[l]}
\end{array}\right] \in \mathbb{R}^{n^{[l]} \times n^{[l-1]}}
$$

to represent the baseline values, which are usually randomly generated within a [-1, 1] interval. The less complex bias vectors can be represented by a one dimensional version of the matrix seen above. In addition, the weighted sum (aka. the values parsed through the activation function) is

$$
z=\sum_{i=1}^{n} x_{i} w_{i}+b
$$

The weighted input can be obtained and parsed through $\mathrm{A}(\mathrm{x})$ for the intermediate column vector

$$
z^{[l]}=\left(\begin{array}{c}
z_{1}^{[l]} \\
z_{2}^{[l]} \\
z_{3}^{[l]} \\
\cdot \\
\cdot \\
\cdot \\
z_{n}^{[l]}
\end{array}\right) \in \mathbb{R}^{n^{[l]}}
$$

The testing model comprised an initial flattening layer, six hidden layers, and one output layer. The flattening layer manipulated the image data into a one-dimensional array for the next layer. The six hidden layers used one of the four activation functions tested and contained between 32-128 layers each. The output layer was always ten neurons, because MNIST, and CIFAR-10 both have ten classes. It used SoftMax instead of a Sigmoid, as probability of classification was distributed between more than two classes. The models trained using SCC loss and the Adam optimizer, which combines aspects of the previously engineered AdaGrad and RMSProp methods. 